\title{
Clinical and Complementary Diagnosis of Sinus Neoplasia in Horses
}

\author{
Tatiana Yumi Mizucina Akutagawa $\left.{ }^{1}\right)^{1,}$ Ricardo de Francisco Strefezzi ${ }^{2}{ }^{2}$, Carla Bargi Belli ${ }^{1}$ ', \\ Raquel Yvonne Arantes Baccarin (iD) ', Luis Cláudio Lopes Correia da Silva (iD) ', André Luis do Valle de Zoppa (iD)', \\ Julio David Spagnolo (iD) ' \& Rodrigo Romero Corrêa (iD)
}

\begin{abstract}
Background: Sinus neoplasms are reported as low frequency in horses. Its clinical characteristics are often nonspecific, depend on complementary methods for diagnosis, and when diagnosed, generally they are already advanced, limiting therapeutic possibilities. The objective of this case series was to detail clinical aspects and complementary exams for sinus neoplasms for early diagnosis, comparing them with the literature.

Cases: Four horses were treated at the College of Veterinary Medicine and Animal Science of the University of São Paulo, with different clinical signs and previous diagnoses, which when evaluated by respiratory endoscopy, radiography, oral cavity evaluation and histopathological exams, revealed the diagnosis of anaplastic carcinoma, poorly differentiated carcinoma, ossifying fibroma and lymphoma. Previous diagnosis, based mainly on clinical signs, were sinusitis secondary to apical infection, ethmoidal hematoma, sinus cyst and sinusitis secondary to periapical disease. The cases of anaplastic carcinoma (case 1) and lymphoma (case 4) presented with an advanced degree of the disease with involvement and destruction of paranasal structures and adjacent tissues, in addition to a poor general condition, which made surgical treatment impossible and led to euthanasia. In these cases, previous treatment was made to apical infection and periodontal disease with secondary sinusitis, but negative evolution led to suspicion of neoplasia, confirmed by histopathological exam of incisional biopsy of the mass in oral cavity. Benign ossifying fibroma (case 3) presented with progressive respiratory difficulty due to occlusion of the lumen of some nasal meatus and radiographic signs of invasion and deformation of the adjacent bones, it was submitted for surgical excision and there was no recurrence until hospital discharge. The poorly differentiated carcinoma (case 2) was a multilobulate neoformation in the ethmoidal region, similar to ethmoid hematoma in endoscopic and radiographic evaluation, it was submitted for excision and two sessions of electrochemotherapy with bleomycin, associated with administration of piroxicam, which obtained a good result until the period of 1 year after discharge.

Discussion: It was found that many characteristics are common with these types of neoplasms, and the clinical signs, such as nasal secretions, airway obstruction, increased facial volume, severe alterations in oral cavity, although unspecific, suggest the differential diagnosis for neoplasms. It is important to differentiate from other diseases noting the evolution and growth of these tumors, like in cases 1 and 4, especially the growth to internal tissues, using complementary methods described here, like endoscopic and radiographic examination. Late identification or even manipulation of neoplasms, without proper diagnosis, leads to a few prognoses regarding life. When it is possible to perform surgical excision, complementary methods are important to guide the procedure, and definitive diagnosis is made through histopathologic exam and some need immunohistochemistry analysis. Cases 2 and 3 had surgical access, were submitted to excision and treatment with good results, both with close monitoring in first months and prolonged quality of life. These results highlight the importance of complementary methods for early diagnosis, correct intervention and monitoring of evolution.
\end{abstract}

Keywords: sinus neoplasia, paranasal sinus, tumor, head, horses. 


\section{INTRODUCTION}

Neoplasms of paranasal sinus are conditions that are difficult to characterize regarding incidence, since it is not possible to affirm how many of these cases are actually recorded [10]. Surveys of cases of sinusitis treated in reference hospitals report $2 \%$ to $19 \%$ of them are related to neoplasm [5,8,9,14,25]. Despite their low incidence, they are challenging affections due to difficult early identification because of their generally insidious onset and prolonged evolution [23]. They show nonspecific clinical signs, and when diagnosed, they are already advanced, limiting therapeutic possibilities. In a review of literature of 50 papers reporting on sinonasal tumors, carried out in 1999 by Head \& Dixon [10], several neoplastic types were identified, with 17 cases of squamous cell carcinoma, 6 adenocarcinomas and 4 lymphomas. Its origin is rarely defined at the time of diagnosis, its growth occurs by expansion and, in malignant cases, infiltration. In these cases they extend to adjacent structures, leading to invasion and bone destruction of the paranasal, nasal cavity and often oral structures. The aim of this series of cases is to report 4 types of sinus neoplasms, detailing the clinical findings and complementary tests that led to the diagnosis in order to add similar characteristics of each of them to the literature and assist professionals in the earlier detection of the disease.

\section{CASES}

The cases presented were attended to at the Veterinary Hospital (Cases 1, 2 and 3) and at the Equine Dentistry Center (Case 4), both in College of Veterinary Medicine and Animal Science (FMVZ) of the University of São Paulo (USP).

Case 1. A 16-year-old American Trotter stallion, weighing $370 \mathrm{~kg}$, with a history of unilateral nasal secretions during 2 months, was attended to by a veterinarian who diagnosed apical infection and secondary sinusitis. Intraoral extraction of the upper left molars (Triadan 209, 210 and 211) was easily performed, and it was noted that they already had intense mobility. After the exodontia, the animal continued to have the nasal secretions and increased facial volume in the maxillary and left submandibular region. In addition, intense weight loss was presented during this period. At the Veterinary Hospital, the body condition score of 3/9 (Henneke Body Condition Scoring System), was apathetic, with increased volume in the left maxillary region and the appearance of local bone loss (Figure 1A). It presented bilateral and purulent nasal secretion in the left nostril, and in a small amount and serous on the right, in addition to halitosis and increased volume of the left mandibular lymph nodes. Due to clinical signs and aggressive evolution, neoplasia was suspected in paranasal sinus. The patient was submitted to dental evaluation and complementary imaging and histopathological examinations.

Under sedation, a dental evaluation was performed, in which the absence of Triadan 209, 210 and 211 was observed. Their respective alveoli were filled with exuberant and necrotic granulation tissue, friable and easy bleeding, as well as ulceration in the palatine region extending to the region of the third and fourth premolars (Triadan 207 and 208). Fine needle aspiration of the neoformation and of the left submandibular lymph node was performed for cytology. In addition, intraoral incisional biopsy of the same region was performed for histopathological examination. Partial obstruction of the airways was found in the endoscopic examination and it was not possible to progress the flexible endoscope through the left nostril. Through the right nostril, no changes were observed in nasal meatus, the newly formed ulcerated appearance was seen occupying the rostral region of the soft palate. With the animal still under sedation, radiographic evaluation was performed, in which increased radiopacity was observed in the alveoli of 209, 210 and 211, invading the maxillary sinuses, with an irregular aspect and with extensive distortion and lysis of the adjacent bones (Figure 1B). In addition, a fluid line was identified in the left paranasal sinus. No more evident alterations were identified. Other evaluations were performed in search of signs of metastasis, such as rectal palpation, abdominal and thoracic ultrasound, but no other alterations were found. Abdominal paracentesis was unproductive.

The cytological examination of the neoformation revealed an acute, septic inflammatory process, with atypias of epithelial cells. From the material collected from the left submandibular lymph node, nodal necrosis and/ or metastatic neoplasia was suspected. histopathological examination of the incisional fragment, stained with hematoxylin and eosin $\left(\operatorname{Synth}^{\circledR}\right)^{1}$, revealed the diagnosis of anaplastic carcinoma in the region of the maxillary sinuses, with extensive exudation and necrosis of the surface, featuring a non-encapsulated neoplastic process, with infiltrative growth and moderate cellularity. 


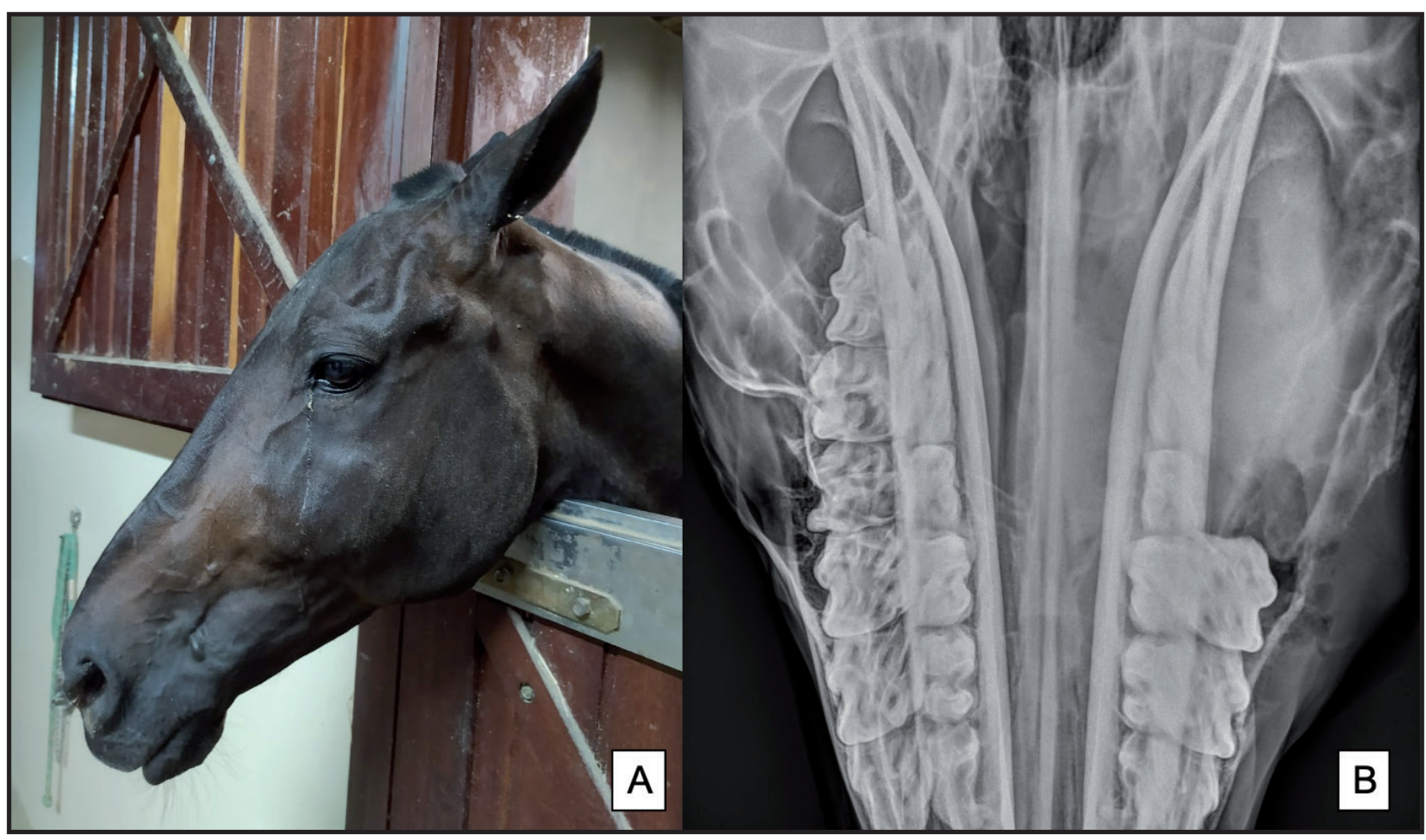

Figure 1. Case 1. Inspection and radiographic evaluation. A- Increased volume in the left maxillary region, palpation showed bone destruction. In the image atrophy of the masseter muscle and epiphore are also observed. B- In the dorsoventral radiographic projection it was possible to observe complete lysis of the left maxillary septum, zygomatic bone and maxilla, in addition to the alveolus of 208 with a radiolucent area around it.

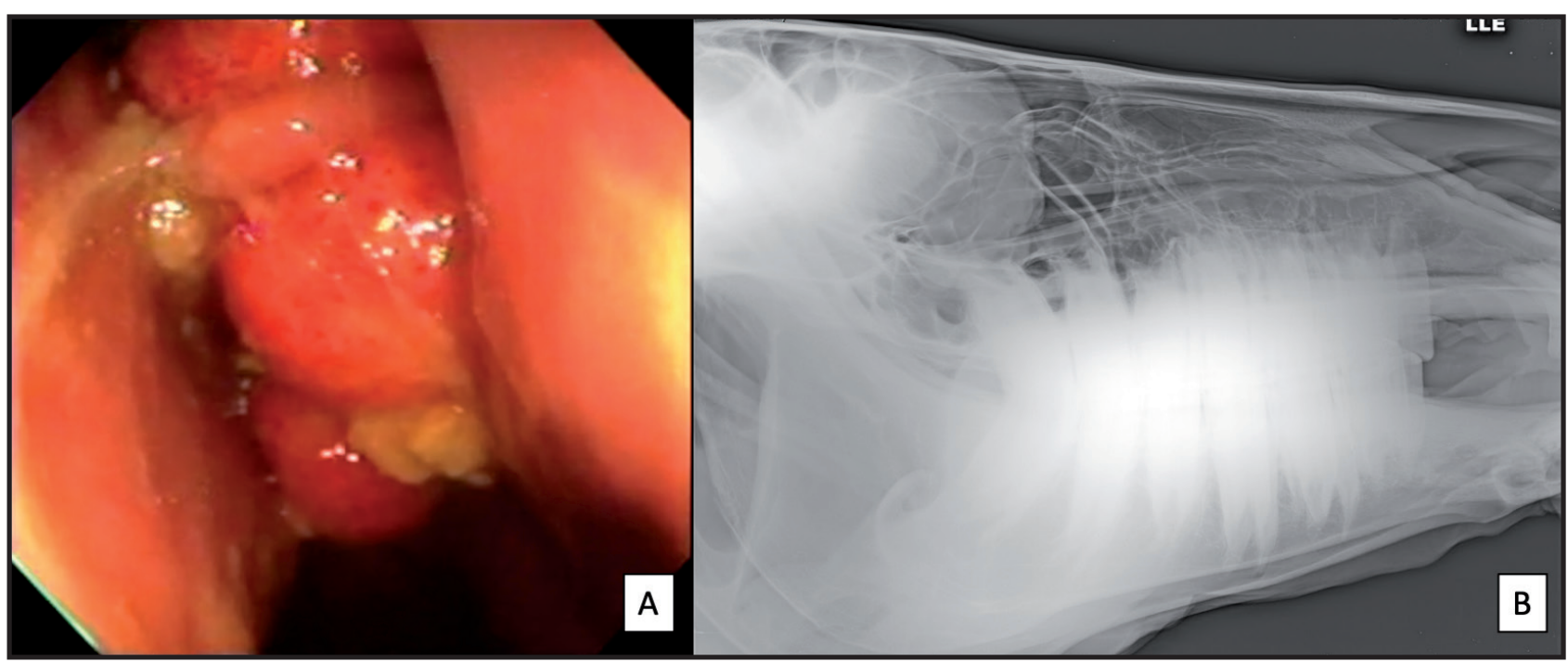

Figure 2. Case 2. Endoscopic and radiographic evaluation. A- Ethmoidal region, evaluated with flexible endoscope, presenting multilobulated neoformation partially obstructing nasomaxillary communication. B- Left lateral-lateral projection evidencing the location of the paranasal radiopaca structure. No dental alterations or areas of lysis and deformation of the bone beams were observed.

Due to the severe involvement of the paranasal sinus and the presence of metastasis, the risks were exposed to the owner and the infeasibility of a surgical intervention was safely described. He chose to remove the animal from the Veterinary Hospital, aware of the clinical picture and poor prognosis regarding life. During telephone contact in the following days, the owner reported that the animal's general condition had worsened; they had started not to feed and were extremely debilitated. The animal died a few days later.

Case 2. A 8-year-old male horse Mangalarga Marchador, weighing $437 \mathrm{~kg}$, with a history of unilateral nasal bleeding after exercise for 2 months, was seen by a veterinarian who identified an ethmoid hematoma through endoscopic evaluation. He was referred to the Veterinary Hospital, presenting in good general condi- 


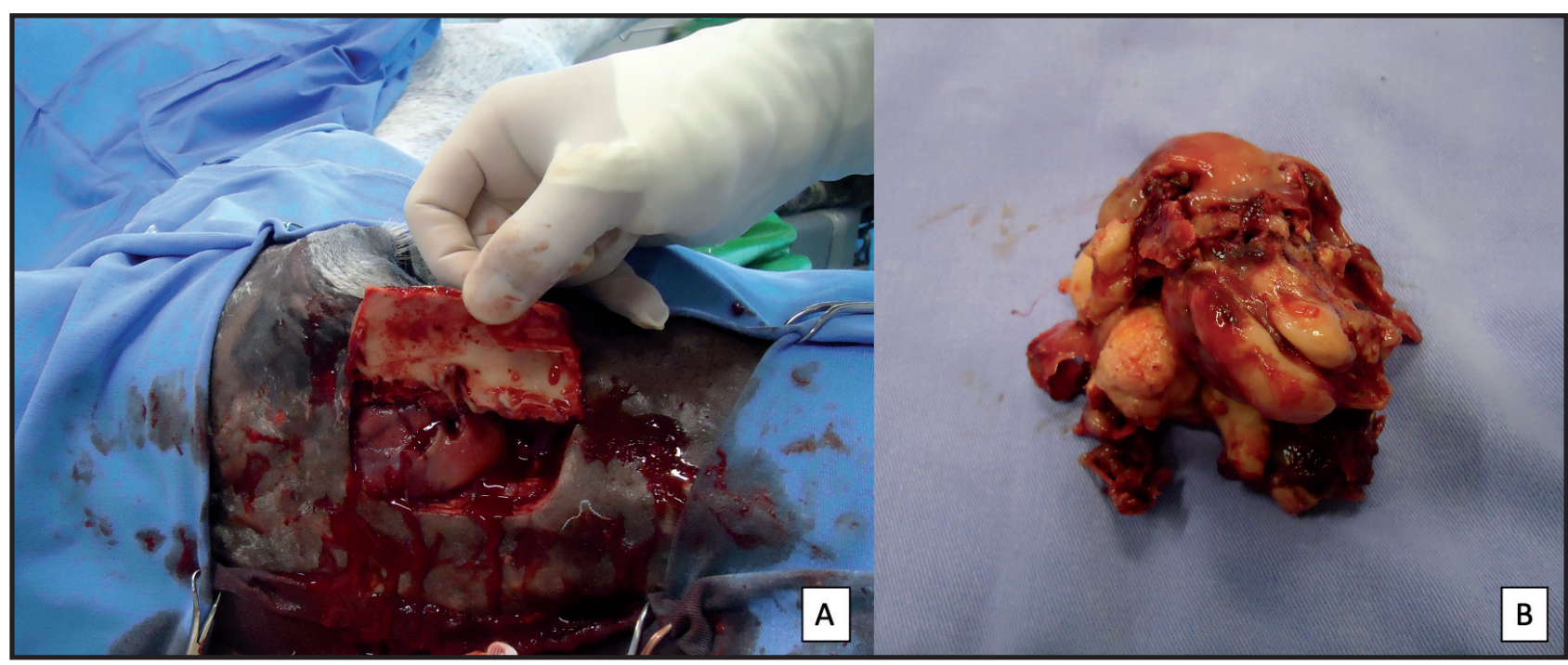

Figure 3. Case 2. Transoperative and neoforming. A- Left frontonasal flap evidencing the neoformation that occupied the frontal sinus and ethmoidal region. B- After excision, irregular appearance of the mass, without defined edges.

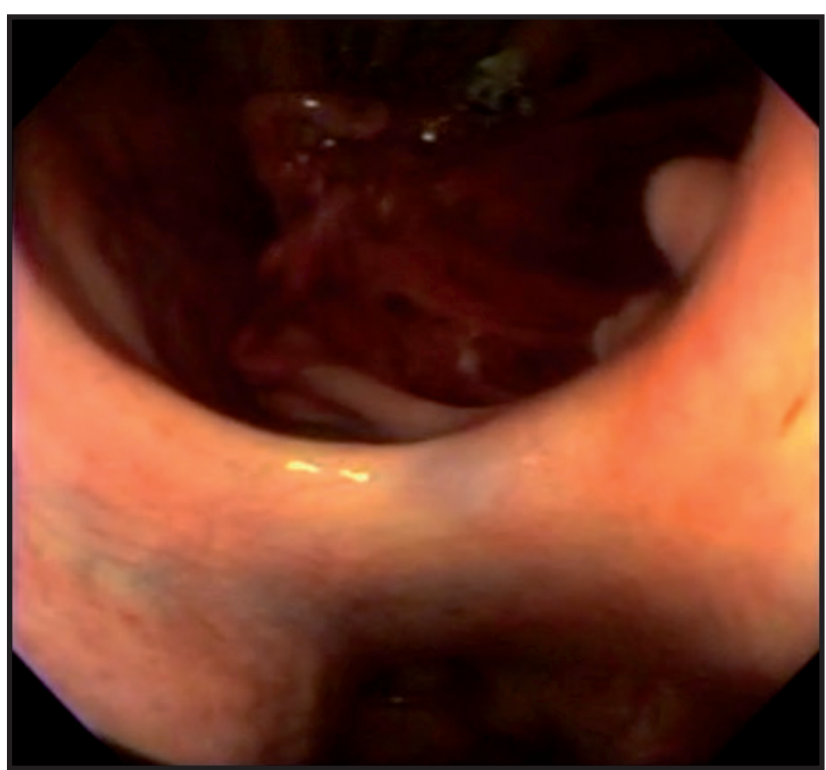

Figure 4. Case 2. Postoperative control rinoscopy. Same area as Figure 2-A, where the neoplasm was visualized. At that time there was direct communication between the frontal sinus and nasal meatus, with destruction of the ethmoturbines.

tion, normal parameters, with purulent secretion in the left nostril and sub-soft sound to the percussion of the maxillary and left frontal sinuses, without increasing the external volume. The submandibular lymph nodes were enlarged and the parotids were palpable.

In the endoscopic evaluation, multilobulate neoformation of hyperemic coloration, covered by mucosa, and with purulent secretion was observed as occupying the caudal aspect of the left middle nasal meatus, in the region of ethmoturbines (Figure 2A). Radiographic images revealed a circumscribed area of increased radiopacity occupying the ethmoidal region, frontal sinus and extending to the left caudal maxillary sinus (Figure 2B).
With the animal under sedation, trephination of the left frontal sinus was performed. Via sinoscopy, the presence of a structure yellowish in color and with a friable aspect was identified, with no defined margins. Multiple fragments were collected by incisional biopsies of the neoformation and sent for histopathological examination. Access was maintained with a Pezzer probe and sinus washes began. Histopathological examination revealed the diagnosis of moderately differentiated adenocarcinoma, however, it was suggested to perform excisional biopsy for analysis of the neoplastic margins and growth pattern.

Under general inhalation anesthesia, left frontonasal sinusotomy was performed. The mass was visualized and manually removed from the inside of the paranasal sinuses (Figure 3). Compressive hemostasis was performed and a gauze roll was placed with its tip in the nasal cavity to control bleeding. The bone flap was repositioned and the skin was sutured. A new trephination of the frontal sinus was performed, caudal to the sinusotomy, and this was maintained with a Pezzer probe. By postoperative sinuscopy, total resection of the mass, loss of definition of the ethmoidal region, presence of yellow to reddish necrotic material in the ethmoidal region and intense vascularization were identified. The ethmoturbinated region was destroyed by the new formation, and there was direct and wide communication with the caudal aspect of the middle nasal meatus.

The histopathological analysis of the obtained material revealed a poorly differentiated malignant epithelial neoplasm, and poorly differentiated adeno- 


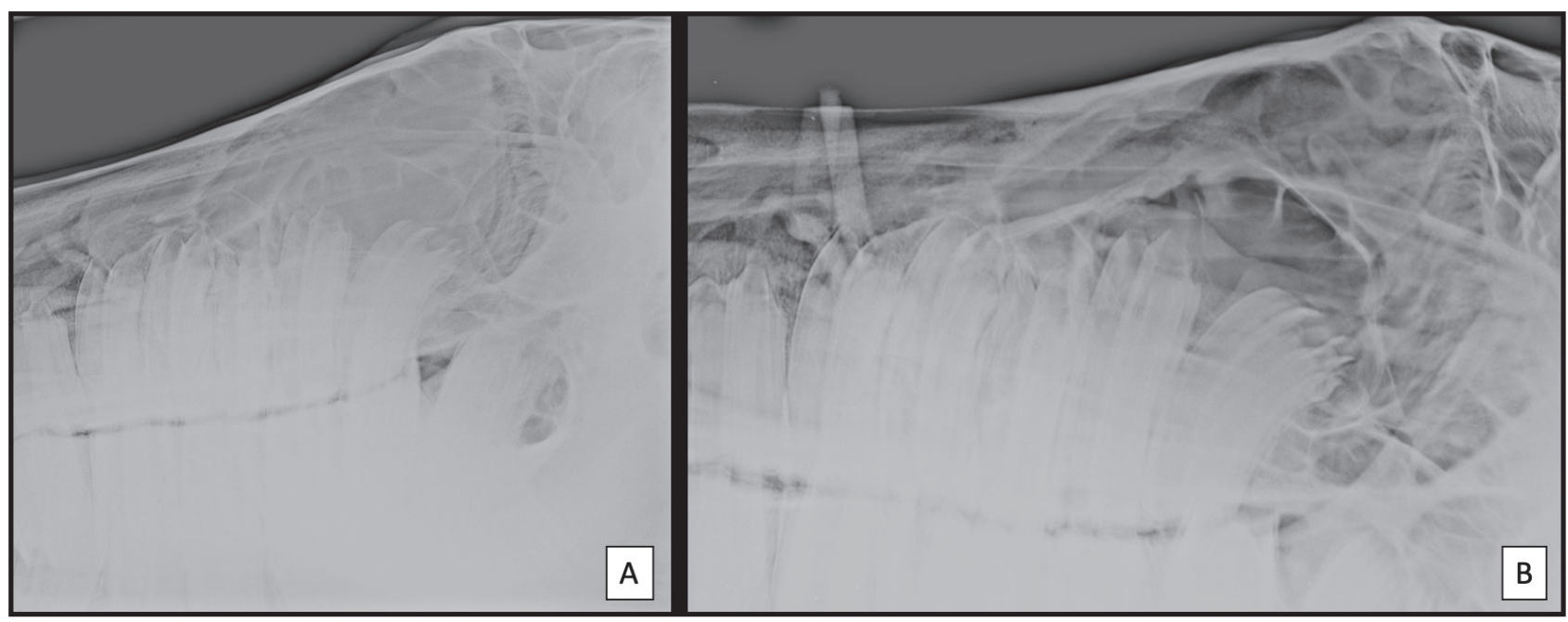

Figure 5. Case 3. Pre- and postoperative radiographs. A- Right oblique dorsoventral projection: the neoformation of the radiopaque characteristic is observed, and inside radiopaque lines similar to a trabecular structure were observed, also occupying the ventral and dorsal conchal sinus. B- Radiographic control 30 days after surgery, where the absence of radiopacity in the right frontal and maxillary sinus is noted.

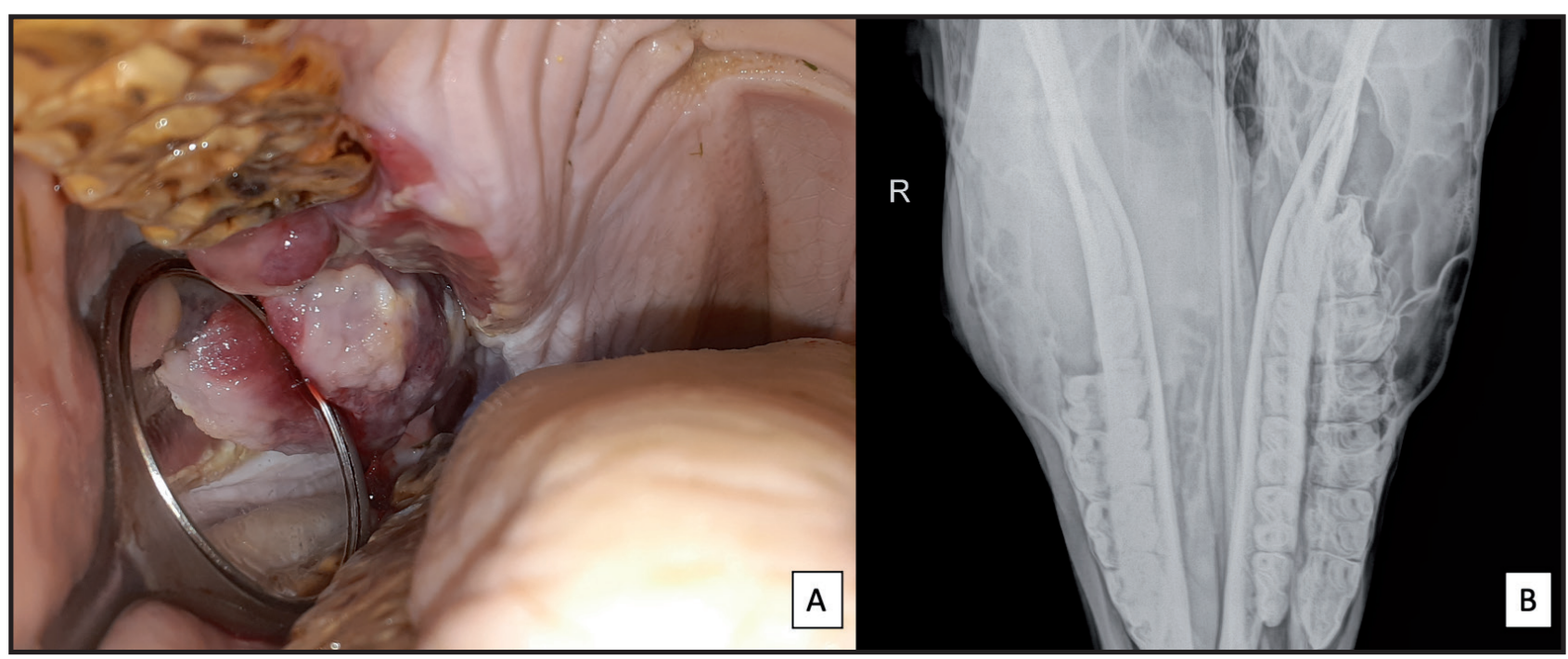

Figure 6. Case 4. Evolution of the neoplasm: oral inspection and radiography. A- Exuberant tissue filling the alveolos of 110 and 111 , approximately 1 month after the exodontias. This same material was collected for histopathological examination. B- Dorsoventral projection: increased radiopacity around the alveoli and in the right maxillary sinuses, with areas of bone lysis and complete loss of bone beams in this region.

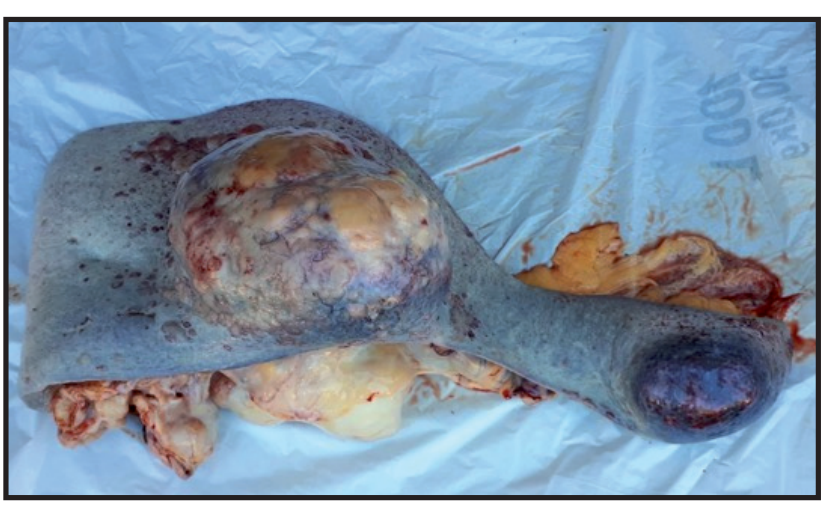

Figure 7. Case 4. Necropsy. Aspect of the spleen presents 2 nodules of different sizes occupying a large part of the parenchyma, one whitish and the other of smaller size at the end of the organ. carcinoma or poorly differentiated squamous cell carcinoma were pointed out as diagnostic hypotheses. The neoplasm presented an infiltrative growth pattern, with foci of bone lysis, non-encapsulated and little delimited. Due to the low degree of differentiation and intense anaplasia, it was suggested that immunohistochemical examination should be performed to determine tumor histogenesis; however, this was not performed.

Ten days after surgical excision, when the diagnosis of the neoplasm was diagnosed, electrochemotherapy with bleomycin was performed. The procedure was performed under general anesthesia and guided by 
sinoscopy by frontal access, infiltrating regions of the tumor aspect still present in the frontal sinus. After 15 days, the second session was performed, following the same anesthetic and surgical protocol. A good healing aspect of the mucosa was observed, presenting intact and without granulomatous proliferations. In the other areas of the paranasal mucosa, no alterations were identified. Flexible respiratory endoscopy revealed direct communication of the nasal cavity with the frontal sinus, in a region where the neoplasm was previously located (Figure 4). One month after electrochemotherapy, no recurrences of neoplasia were observed. On control radiographs there was a significant reduction in tissue radiopacity, but it was still present in the same regions. In this examination, the ethmoturbines and maxillary septum could not be identified.

Throughout the hospitalization period, the animal was maintained with a Pezzer probe, through the frontal accesses, for sinus washes, twice a day. Piroxicam administration [ForVets ${ }^{\circledR 2}-0.2 \mathrm{mg} / \mathrm{kg}$, PO, SID]started 1 month after the first electrochemotherapy section, and the treatment was maintained for 49 days, until medical discharge. The patient was discharged after 4 months of hospitalization, with no signs of recurrence or metastasis. One year after discharge, however, the animal died with neurological signs.

Case 3. A 4-year-old castrated male Brazilian Equestrian horse, weighing $515 \mathrm{~kg}$ presented progressive respiratory difficulty with an evolution of 5 months, initially diagnosed as laryngeal hemiplegia. He also presented respiratory noise during exercise and began to present bilateral nasal secretions of purulent aspect. The day before referral, a veterinarian repeated the respiratory endoscopy and suggested the diagnosis of bilateral sinus cyst. At the Veterinary Hospital, the patient was in good general condition, but presented bilateral purulent nasal secretions, crackle at bilateral thoracic auscultation, and submandibular lymph nodes with firm consistency.

Left rhinoscopy showed bulging of the nasal septum and dorsal nasal concha, causing a decrease in the lumen of the dorsal and common meatus, in addition to the presence of a small amount of serous secretion. Right rhinoscopy showed bulging of the ventral shell and severe deformity of the dorsal shell, with total occlusion of the lumen of the dorsal, middle and ventral meatus. Radiographic examination identified a circumscribed radiopaque area, with an irregular surface, occupying the right frontal and maxillary sinuses with invasion and deformation of the adjacent bones (Figure 5A) and deviation of the nasal septum to the left.

Under general inhalation anesthesia, a right maxillary sinusotomy was performed. A large amount of fibrous tissue was removed, both manually and with the aid of hemostatic forceps. Compression hemostasis was performed and roll gauze was inserted with topical povidone iodine $\left(\text { Riodeine }^{\circledR}\right)^{3}$ for removal in $72 \mathrm{~h}$. Two Pezzer probes $\left(\text { Well Lead }{ }^{\circledR}\right)^{4}$ were fixed, one in each frontal sinus, accessed by trephination, in addition to a probe in the right maxillary sinus, for sinus washes. The periosteum, subcutaneous tissue and skin were sutured.

The collected material was submitted for histopathological examination, which concluded the diagnosis of ossifying fibroma. Microscopic evaluation revealed proliferation of the fusiform cells, with moderate anisocytosis and anisocariosis. The cells often involved mineralized bony trabeculae, surrounded by multinucleated giant cells. In the midst of proliferation, intensely vascularized loose collagenous stroma was observed, with foci of hemorrhage, hemosiderin deposits and foci of lymphoplasmacytic inflammatory infiltrate.

Sinus washes were performed with $2 \mathrm{~L}$ of $0.9 \%$ saline solution (Fresenius $\left.\mathrm{Kabi}^{\circledR}\right)^{5}+$ topical povidone iodine (Riodeine ${ }^{\circledR 3}-0.1 \%$ ), for each probe installed, twice a day. Rhinoscopy and sinoscopy were performed 1 week after surgery. There was still a decrease in the lumen of the ventral and middle meatus in the left nasal cavity due to the persistence of fibrous tissue in the ventral and dorsal concha. In the right nasal cavity there was little fibrous tissue in the recess of the ventral and dorsal nasal concha, and also a considerable amount of material in both shells. The evaluation was repeated 30 days after surgery, when a progressive reduction in nasal secretions was noticed. A discrete bulging of the nasal septum and dorsal concha was observed, allowing the free passage of the endoscope through the left nasal cavity. On the right side there was still lumen occlusion, however, the deformities were less severe than in previous exams. Upon radiographic examination, there was a significant reduction in radiopacity in the frontal and maxillary sinus, with bone distortion inside them (Figure 5B).

The animal was discharged after one week, with recommendations to maintain the sinus washes with $2 \mathrm{~L}$ of $0.9 \%$ saline solution + topical PVPI $0.1 \%$ 
until control of the nasal secretions had been achieved. A return was scheduled in 30 days for reassessment, but this was not met by the owner.

Case 4. A 22-year-old male equine with no defined breed, weighing $420 \mathrm{~kg}$, presented unilateral nasal secretions 1 month ago. It was treated on the property with antibiotic therapy without result. Dental evaluation was performed where changes in the periodontium were observed in the region of the second and third upper and right molars (teeth 110 and 111), with gingival and palatal ulceration, in addition to severe tooth mobility. Radiographic examination showed changes in these same dental elements, in addition to sinusitis. The patient was then referred to the Equine Dentistry Center at FMVZ-USP. Good general condition was noted. Clinically, there was a large amount of secretion in the right nostril, with a foul odor and purulent aspect, in addition to the absence of air flow in the same nostril. Initially, sinusitis secondary to periodontal disease was suspected in 110 and 111 .

Endoscopic evaluation showed no changes in the nasal structures, with secretions only being drained by the nasomaxillary opening. Radiographs showed increased radiopacity in the right maxillary sinuses, with loss of definition of the maxillary septum and alveoli of 110 and 111; these same dental elements showed root remodeling and periapical halo. The dental physical examination and oroscopy were repeated, allowing the visualization of palatal ulceration in the regions of 110 and 111 , with severe mobility of both dental elements, which was characterized as severe periodontal disease. There was exuberant and pinkish granulation tissue on the buccal and distal gingival margin of 111. On tooth 108 there was a palatal and mesial periodontal fistula, with drainage of purulent secretions. The millimeter exploration probe could be fully introduced, and moderate mobility was noted in this tooth.

In the same procedure, with the animal under sedation, intraoral exodontia of 110 and 111 was performed, with great ease due to the severity of the periodontal disease. An extensive oroantral fistula was identified in both alveoli, which were occluded with a polyvinylsiloxane plug (PVS). Then trephination of the right caudal and frontal maxillary sinus was performed, where there was immediate drainage of purulent and thick secretions from the frontal access. Fixation of a Pezzer probe was performed in both accesses for subsequent sinus washes with $0.1 \%$ topical povidone iodine solution $\left(\text { Riodeine }^{\circledR}\right)^{3}$

Despite the treatment used, healing of the alveolus was not observed in the dressings performed. There was a proliferation of exuberant tissue that extended towards the palate and vestibule (Figure 6A). Initially, alveolar dressings were performed every 2 days, with pressure washing and alveolar debridement. However, in 30 postoperative days, the animal presented an increase in hot and painful volume on palpation in the region of the right masseter, which extended to the maxillary region. The most floating point was lanced when an abscess was suspected, but there was no drainage of purulent secretions. The ultrasound evaluation showed a heterogeneous structure, without precise delimitation and which was not consistent with abscess formation, besides the marked edema. Neoplasia was suspected, and biopsy of the granulation material present in the alveoli was performed for histopathological examination.

The diagnosis of large cell lymphoma was obtained. Extensive proliferation of the round cells was observed, which were arranged in large groups amidst the delicate fibrovascular stroma. The nuclei of the neoplastic cells were large $(>2 x$ the diameter of a red blood cell), round, and with some with contour irregularities (indentations). They showed irregular chromatin, with multiple nucleoli in most cells. There were a large number of mitoses ( $>10$ per field), with the presence of atypical mitoses Immunohistochemistry was suggested to differentiate the cell type, however, it was not possible to complete the exam. After diagnostic confirmation, the presence of metastases was investigated. An abdominal ultrasound revealed a nodule of approximately $12 \mathrm{~cm}$ located in the spleen, which is, therefore, a multicentric lymphoma. Control radiography showed formation of the right maxillary sinuses more clearly, in addition to more pronounced bone lysis (Figure 6B).

One month after hospitalization, the animal's general condition worsened, and they started to show hyporexia and progressive weight loss. In common agreement with the owner, euthanasia was performed. At necropsy, it was possible to observe the nodule in the spleen, previously identified on the abdominal ultrasound, occupying a large part of the parenchyma (Figure 7). No other changes were observed in the other systems. 


\section{DISCUSSION}

Among sinus neoplasms, it is suggested that squamous cell carcinoma is the most commonly observed type [14,17], with its origin being both the paranasal sinuses and the oral cavity $[3,10]$. In case 1 , it was not possible to define the primary site, as both were affected. Glandular epithelial tumors are more common in the ethmoid and frontal region, possibly due to the presence of Bowman's glands in the ethmoid $[3,14]$. This characteristic supports the diagnostic possibility of poorly differentiated adenocarcinoma in case 2, instead of squamous cell carcinoma, which is more common in the maxillary sinuses [10]. Of the cases presented, only the ossifying fibroma (case 3) is classified as benign, despite there being masses of expansive growth and that it can progressively destroy adjacent bones [14]. The affected animals are generally young [14], as in case 3, and diagnosed at 4 years of age. Lymphoma cases (case 4) are not predisposed to age, race or gender $[1,14,24]$. It is the most common neoplasm of the hematopoietic system of horses, originating from peripheral lymphoid tissues, with a malignant and aggressive characteristic [14,22,23].

The evolution time of the neoplasms presented varied from 1 to 5 months before being referred to the Veterinary Hospital, corroborating the average of 70 days reported by Dixon et al. [5]. Clinical signs are generally nonspecific and similar to those of chronic sinusitis, such as unilateral nasal discharge, usually purulent, which may progress to bilateral with tumor growth $[3,5,25]$. All cases presented with purulent nasal discharge, being bilateral in 3 , with a longer evolution time. There are few reports of epistaxis [8], which was initially observed in case 2, where it is suggested to be due to the involvement of the ethmoid. Increases in facial volume are common, usually firm, with no pain on palpation and greater when compared to sinusitis of dental origin [5]. In cases 1 and 4, there was a large increase in volume with bone remodeling and rapid evolution. Two of them also had difficulty breathing, due to the intraluminal growth of the mass that led to the obstruction of the air flow in the nostrils and even deviation of the nasal septum, which was frequently observed between 40 and 50\% [3,5]. Signs of halitosis and bad chewing, in addition to severe emaciation, were observed in cases where there was involvement of the oral cavity. Due to nonspecific clinical signs and limited diagnostic methods available before referral, there was no initial suspicion of neoplasia in most cases, with treatment being performed on the property without diagnostic confirmation.

One of the complementary methods used before referral is respiratory endoscopy. Direct visualization of the neoplasm was possible in 13 out of 20 cases reported by Dixon et al. [3]; the exam is also useful when there are deformities in the upper airways [23]. Cases of adenocarcinoma often present as lobulated masses in the ethmoid region, in a typical location of ethmoid hematoma $[8,11]$, as was initially suspected in case 2. Reports of ossifying fibroma, as observed in the present case, present with historical frequency of noise and difficulty breathing $[15,20]$. At endoscopy, airway obstruction is observed, preventing progression of the endoscope, as in case 3, since its expansive growth leads to deformation of the nasal conchae.

Radiographic examination is also useful when endoscopy is not available [3] for the determination of location and possibly affected tissues [23]. It is not possible to differentiate the types of tumor through radiographic characteristics, but some aspects are coincident with the literature, such as the trabecular structure observed in case 3, similar to a report of ossifying fibroma [15], and the alterations found in anaplastic carcinoma, significant lysis of adjacent bones and loss of definition of dental alveoli [16]. It is important to consider that cases of sinus neoplasia show more aggressive radiographic changes when compared to sinusitis secondary to dental changes or non-neoplastic masses, and this must be a differential diagnosis when faced with these images [7]. Computed tomography has become a viable tool in reference centers, as it is important to provide information on the exact location of the mass, guiding the indication and surgical planning [21].

In cases where the neoplasm extends into the oral cavity, it is important to inspect the animal under sedation. Lesions similar to case 1 (anaplastic carcinoma) are described in the literature, such as necrotic lesions on the hard palate, an ulcerated mass near the vestibular teeth and oral hemorrhage $[3,17]$. Depending on the degree of evolution, these changes can lead to mobility and tooth loss. In case 4, dental changes were detected before the suspicion of neoplasia, being initially diagnosed as periodontal disease and secondary sinusitis. The degree of involvement of the alveolar structures and the worsening of the condition after the start of treatment 
led the authors to investigate the nature of the lesion and then to diagnose large cell lymphoma.

The definitive diagnosis is made through histopathological examination of the lesion [23], and in some cases immunohistochemical examination is necessary for confirmation. In case 1 , mass cytology and submandibular lymph nodes were also performed, with cellular atypia suggestive of a neoplastic process being observed. Due to limited access to the paranasal sinuses, in some cases an incisional or transendoscopic biopsy is performed, however, the small sample size and its often superficial nature may not be representative of the entire lesion, making it difficult to characterize it [3]. For example, case 1 was diagnosed as anaplastic carcinoma based on the analysis of a fragment collected through the oral cavity. It was not possible to undergo an excisional biopsy, therefore, it cannot be said that this would be the histological pattern of the entire tumor. The extent and degree of involvement of the other structures limited the diagnosis in this case. In case 2, it was possible to observe the contrast of the 2 forms of collection, through incisional biopsy, which revealed moderately differentiated adenocarcinoma, and excisional biopsy, which revealed two diagnostic hypotheses, including poorly differentiated adenocarcinoma or poorly differentiated squamous cell carcinoma, due to the low grade cell differentiation observed in the sample as a whole.

For excisional biopsy, the ideal collection method is performed through access to paranasal sinuses by trepanation or bone flap [23], as was done in case 3 . Its histological characteristics were similar to reports of ossifying fibroma in the paranasal sinuses, as proliferation of spindle cells in combination with collagen fibers and expansive growth, with destruction of bone trabeculae accompanied by multinucleated giant cells $[15,20]$. Some cases require more than one diagnostic method for the complete neoplastic classification, such as case 4, which was diagnosed as large cell lymphoma. According to the classification of the World Health Organization, it can be subdivided into 14 subtypes according to cell characteristics, being B, T or mixed cells, and for that it would be necessary to perform an immunohistochemical examination [6].

The investigation of other systems in search of signs of metastasis is of paramount importance during diagnosis and especially in the determination of prognosis, since it is the reason why surgical excision can fail around $50 \%$ of patients [18]. In these cases, surgical removal becomes palliative, in order to improve the quality of life of the animal [14]. Of the cases presented, except for case 3, of a benign nature, all could spread throughout the body. In case 1 , the possibility of metastasis was raised because of the cytological alterations observed in the submandibular lymph node; despite the invasive growth of carcinomas, metastases are rare, and when they occur, they are usually observed precisely in adjacent lymph nodes [14]. In case 4 , a nodule in the spleen was observed by abdominal ultrasound, indicative of metastasis and consistent with alterations found in 6 of 13 horses with lymphoma by Janvier et al. [12]. In case 2 no signs of metastasis were detected, which may be related to longer survival time after treatment in relation to the others.

The importance of imaging tests is also highlighted in the treatment and monitoring of the evolution of these cases. Among the therapeutic options, surgical excision aims at the total removal of the neoplasia, which is a challenge due to the difficulty of defining safe margins in the paranasal sinus region, which can make removal impossible due to the affected structures $[13,14,17]$. Case 1 was in an advanced stage at the time of diagnosis, with evident destruction of the facial bones and internal structures, airway obstruction and growth in the oral cavity, with loss of dental elements. The animal still had a low body condition score, with marked difficulty in feeding. Considering the patient's surgical impossibility and clinical condition, euthanasia was indicated.

Surveys of malignant neoplasms treated exclusively with surgical resection commonly lead to an even more aggressive relapse, leading to the need for euthanasia [3]. The combination of therapies is an alternative to offer a better prognosis, such as a combination of chemotherapy, mainly topical or intralesional [14], radiotherapy, which presents satisfactory results in horses $[7,20,26]$ or electrochemotherapy. In case 2 , surgical excision was performed and two electrochemotherapy sessions were associated with bleomycin. This method involves the intralesional administration of chemotherapy, followed by the application of electrical pulses through the tumor, which increases the entry of the agent into the cells through electroporation, increasing its cytotoxicity and consequently the effectiveness of the treatment, in addition to local action altering blood flow in tumor tissue [2]. There are 
no prior reports of the use of electrochemotherapy in paranasal neoplasms in equines. In the case described, since it was not possible to perform surgical excision with safe margins due to its location, the combination of electrochemotherapy was positive, and prolonged the time and quality of life of the animal. No relapse of the lesion was observed until the end of the period of hospitalization ( 2 months), and the animal remained without clinical signs according to the owner for one year. The use of piroxicam for 49 days was also associated. There are few reports of its use in horses and its exact mechanism has not yet been elucidated, however, since prostaglandins derived from COX-2 (Cyclooxygenase type 2) may be responsible for tumor growth, angiogenesis and metastasis, it is believed that COX-2 inhibitors may act by inhibiting these processes [19]. The treatment was effective in prolonging and providing quality of life to the animal, however, this was dead after a period of 1 year since presenting neurological signs. Necropsy could not be performed, but the sudden onset of signs suggests metastasis in the central nervous system.

Despite the therapeutic options presented, most cases in the literature $[4,9]$ and in the present series of cases are diagnosed in an advanced degree of tumor growth, in which there is destruction of the paranasal sinus, limiting the surgical possibilities and associations of therapies. It is not possible to affirm how much manipulation prior to the correct diagnosis can contribute to the evolution of the neoplasm. It is clear from these reports that cases of sinus neoplasms are challenging in the early identification aspect, and that early diagnosis of them is a determinant to enable surgical intervention and control of neoplastic growth.

It was possible to identify common characteristics of certain types of sinus neoplasia, which, despite being nonspecific signs, may suggest the differential diagnosis. It is common for clinical signs to be associated with more frequent changes, such as sinusitis secondary to dental alterations or non-neoplastic masses, such as ethmoidal hematoma or sinus cysts. However, it is important to note the evolution and growth of these tumors, especially in internal tissues, and it is in this aspect that the importance of performing complementary methods for diagnosis lies. Late identification or even manipulation of neoplasms, without proper diagnosis, leads to a few prognoses regarding life.

\section{MANUFACTURERS}

${ }^{1}$ Labsynth Produtos para Laboratórios Ltda. Diadema, SP, Brazil.

${ }^{2}$ Forvets Farmácia De Manipulação Veterinária, Comércio e Serviços Ltda. São Paulo, SP,Brazil.

${ }^{3}$ Rioquímica S.A. São José do Rio Preto, SP, Brazil.

${ }^{4}$ Well Lead Medical Co. Ltd. Guangzhou, GD, China.

${ }^{5}$ Fresenius Kabi Ltda. Aquiraz, CE, Brazil.

Declaration of interest. The authors report no conflicts of interest. The authors alone are responsible for the content and writing of paper.

\section{REFERENCES}

1 Aleman M. \& Watson J.L. 2015. Lymphoma in horses. In: Smith B.P. (Ed). Large animal internal medicine. 5th edn. St. Louis: Elsevier, pp.1073-1075.

2 Brunner C.H.M. 2015. Avaliação do comportamento vascular do tumor de Ehrlich na forma sólida em camundongos submetidos à eletroquimioterapia com bleomicina. 75f. São Paulo, SP. Tese (Doutorado em Anatomia dos Animais Domésticos e Silvestres) - Programa de Pós-Graduação da Faculdade de Medicina Veterinária e Zootecnia, Universidade de São Paulo.

3 Dixon P.M. \& Head K.W. 1999. Equine nasal and paranasal sinus tumours: Part 2: A contribution of 28 case reports. Veterinary Journal. 157(3): 279-294.

4 Dixon P.M., Parkin T.D., Collins N., Hawkes C., Townsend N., Tremaine W.H., Fisher G., Ealey R. \& Barakzai S.Z. 2012. Equine paranasal sinus disease: A long-term study of 200 cases (1997-2009): Treatments and long-term results of treatments. Equine Veterinary Journal. 44(3): 272-276.

5 Dixon P.M., Parkin T.D., Collins N., Hawkes C., Townsend N.B., Fisher G., Ealey R. \& Barakzai S.Z. 2011. Historical and clinical features of 200 cases of equine sinus disease. Veterinary Record. 169(17): 439.

6 Durham A.C., Pillitteri C.A., Myint M.S. \& Valli V.E. 2012. Two Hundred Three Cases of Equine Lymphoma Classified According to the World Health Organization (WHO) Classification Criteria. Veterinary Pathology. 50(1): 86-93.

7 Gerard M., Pruitt A. \& Thrall D.E. 2010. Radiation therapy communication: Nasal passage and paranasal sinus lymphoma in a pony. Veterinary Radiology and Ultrasound. 51(1): 97-101. 
8 Hanna A., Stieger-Vanegas S.M., Heidel J.R., Esser M., Schlipf J. \& Mecham J. 2015. Nasal adenocarcinoma in a horse with metastasis to lung, liver, and bone and review of metastasis in nine horses with sinonasal tumors. Case Reports in Veterinary Medicine. 2015(4): 1-6. DOI: 10.1155/2015/845870.

9 Hart S.K. \& Sullins K.E. 2011. Evaluation of a novel postoperative treatment for sinonasal disease in the horse (19962007). Equine Veterinary Journal. 43(1): 24-29.

10 Head K.W. \& Dixon P.M. 1999. Equine nasal and paranasal sinus tumours. Part 1: Review of the literature and tumour classification. Veterinary Journal. 157(3): 261-279.

11 Hepburn R.J. \& Furr M.O. 2004. Sinonasal Adenocarcinoma Causing Central Nervous System Disease in a Horse. Journal of Veterinary Internal Medicine. 18(1): 125-131.

12 Janvier V., Evrard L., Cerri S., Gougnard A. \& Busoni V. 2016. Ultrasonographic findings in 13 horses with lymphoma. 57(1): 65-74.

13 Knottenbelt D.C. \& Kelly D.F. 2011. Oral and dental tumors. In: Easley J., Dixon P.M. \& Schumacher J. (Eds). Equine Dentistry. 3rd edn. Edinburgh: Elsevier, pp.149-181.

14 Knottenbelt D.C., Patterson-kane J.C.\& Snalune K.L. 2015. Squamous cell carcinoma. In: Clinical Equine Oncology. Edinburgh: Elsevier Ltd, pp. 220-236.

15 Kodaira K., Muranaka M., Naito Y., Ode H., Oku K., Nukada T. \& Katayama Y. 2010. Histopathological characteristics of an ossifying fibroma formed in the maxilla of a racehorse. Journal of Equine Science. 21(1): 7-10.

16 Kowalczyk L., Boehler A., Brunthaler R., Rathmanner M. \& Rijkenhuizen A.B.M. 2011. Squamous cell carcinoma of the paranasal sinuses in two horses. Equine Veterinary Education. 23(9): 435-440.

17 Łopuszyński W., Bulak K., Komsta R., Twardowski P. \& Kusy R. 2018. Thyroid Metastases From Sinonasal Squamous Cell Carcinoma of a Horse - A Case Report and Literature Review. Journal of Equine Veterinary Science. 67: 31-36.

18 Mair T.S. \& Couto C.G. 2006. The use of cytotoxic drugs in equine practice. Equine Veterinary Education. 18(3): 149-156.

19 Moore A.S., Beam S.L., Rassnick K.M. \& Provost P. 2003. Long-term control of mucocutaneous squamous cell carcinoma and metastases in a horse using piroxicam. Equine Veterinary Journal. 35(7): 715-718.

20 Orsini J.A., Baird D.K. \& Ruggles A.J. 2004. Radiotherapy of a recurrent ossifying fibroma in the paranasal sinuses of a horse. Journal of the American Veterinary Medical Association. 224(9): 1483-1486.

21 Pease A.P. 2013. The equine head. In: Thrall D.E. (Ed). Textbook of Veterinary Diagnostic Radiology. 6th edn. St. Louis: Elsevier, pp.153-171.

22 Sano Y., Okamoto M., Ootsuka Y., Matsuda K., Yusa S. \& Taniyama H. 2017. Blindness associated with nasal/ paranasal lymphoma in a stallion. Journal of Veterinary Medical Science. 79(3): 579-583.

23 Scarratt W.K. \& Crisman M.V. 1998. Neoplasia of the respiratory tract. Veterinary Clinics of North America: Equine Practice. 14(3): 451-473.

24 Taintor J. \& Schleis S. 2011. Equine lymphoma. Equine Veterinary Education. 23(4): 205-213.

25 Tremaine W.H. \& Dixon P.M. 2001. A long-term study of 277 cases of equine sinonasal disease. Part 1: Details of horses, historical, clinical and ancillary diagnostic findings. Equine Veterinary Journal. 33(3): 274-282.

26 Weaver M.P., Dobson J.M. \& Lane J.G. 1996. Treatment of intranasal lymphoma in a horse by radiotherapy. Equine Veterinary Journal. 28(3): 245-248. 